\title{
Leader tracking in homogeneous vehicle platoons with broadcast delays
}

\author{
Andrés A. Peters ${ }^{\mathrm{a}, 1}$, Richard H. Middleton ${ }^{\mathrm{b}}$, Oliver Mason ${ }^{\mathrm{a}}$ \\ ${ }^{a}$ Hamilton Institute, National University of Ireland Maynooth, Maynooth, Co. Kildare, Ireland \\ ${ }^{\mathrm{b}}$ Centre for Complex Dynamic Systems and Control, The University of Newcastle, Callaghan NSW 2308, Australia
}

\section{A R T I C L E I N F O}

\section{Article history:}

Received 19 October 2012

Received in revised form

27 May 2013

Accepted 9 September 2013

Available online 18 October 2013

\section{Keywords:}

Vehicle platoons

Leader following

String stability

Time delay

Linear systems

\begin{abstract}
A B S T R A C T
For vehicle platoons, the leader following control architecture is known to be capable of achieving string stability while maintaining tight formations. In this paper, we study a variety of schemes where the leader state is available to the other members of the platoon. We show that in some cases it is possible to achieve string stability in the presence of certain amounts of time delay in the leader state reception. We also compare other properties of the different schemes and discuss some of their advantages and disadvantages.
\end{abstract}

(C) 2013 Elsevier Ltd. All rights reserved.

\section{Introduction}

In recent decades, formation control of autonomous vehicles has received great attention; see for example Chien and Ioannou (1992), Chu (1974), Levine and Athans (1966) and Swaroop and Hedrick (1996). More recently, researchers have studied extensively the simple case of a 1-D platoon of vehicles with linear dynamics, considering diverse alternatives to achieve coordinated movement of the string (see for example Hao, Yin, \& Kan, 2012; Jovanovic \& Bamieh, 2005; Lin, Fardad, \& Jovanovic, 2012, and the references therein).

One simple approach, which can be implemented using linear controllers, is to equip every member of the formation with a compensator that stabilizes its position in closed loop, using as a reference the position of its predecessor in the string and a desired constant inter-vehicle spacing. The internal stability of the whole system is ensured by the simple interconnection, the design of

\footnotetext{
This work was supported by the Irish Higher Educational Authority (HEA) PRTLI 4 Network Mathematics Grant and PRTLI 5 Telecommunications Graduate Initiative Grant. The material in this paper was not presented at any conference. This paper was recommended for publication in revised form by Associate Editor Wei Ren under the direction of Editor Frank Allgöwer.

E-mail addresses: andres.peters@nuim.ie (A.A. Peters),

Richard.Middleton@newcastle.edu.au (R.H. Middleton), oliver.mason@nuim.ie (O. Mason).

1 Tel.: +353017086100; fax: +353017086269.
}

the compensator and the assumption of linear dynamics for each vehicle. In Seiler, Pant, and Hedrick (2004) it was shown that this simplistic architecture suffers from a drawback known as "string instability" namely, the amplification of disturbances along the string as a response to a disturbance in a single vehicle. In this case, the problem occurs if identical vehicles and local controllers are used (homogeneous control) and whenever the controller-vehicle pair has two integrators, regardless of the chosen compensator parameters. Moreover, in Barooah and Hespanha (2005) the authors show that including also the immediate follower position in the control signal of each vehicle (bidirectional control) does not remove the disturbance amplification, complementing the work done in Seiler et al. (2004).

The term "string stability" has been defined in many different ways. In this work we consider a similar approach to that in Middleton and Braslavsky (2010): in an interconnection of multiple systems, we consider a set of relevant closed loop transfer functions (e.g. the ones that describe the effect of disturbance on the inter-vehicle spacings). String stability occurs if the functions have frequency magnitude peaks that are bounded independently of the platoon size. String instability, has a number of undesirable implications for the safety and performance of a platoon of vehicles, the most dramatic being the increased chance of collisions as the size of the platoon grows. Several measures aimed at ensuring string stability of a formation have been proposed. The authors of Chien and Ioannou (1992), Klinge and Middleton (2009) and Swaroop, Hedrick, Chien, and Ioannou (1994) introduce "time headway", 

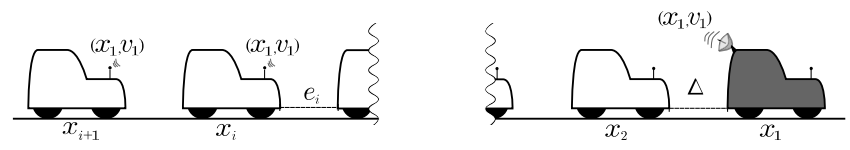

Fig. 1. Platoon of vehicles.

where inter-vehicle spacings are dependent on vehicle velocities. Equipping each vehicle with a controller that depends on its position along the string (heterogeneous control), has also been proposed to overcome the difficulty, see for example Khatir and Davidson (2004), Lestas and Vinnicombe (2007) and Shaw and Hedrick (2007). Unfortunately this only helps if control "bandwidths" are allowed to diverge (either to 0 or $\infty$ ) as the string length grows, (Middleton \& Braslavsky, 2010). Alternatively, "leader following" schemes such as the ones studied in Seiler et al. (2004) and Xiao, Gao, and Wang (2009) obtain string stability of the formation by providing every follower with the state (or an estimate) of the leader, at the cost of increasing networking requirements. A limited range of forward communication, which would imply a loss in leader state reception along the string, does not allow linear controllers to achieve string stability without the use of constant time headway policies (see Middleton and Braslavsky (2010)). The use of a network to provide the members of a string of vehicles with the leader state (position and/or velocity) immediately poses questions on the effect of disruptions of the communication. In this context, the works presented in Liu, Goldsmith, Mahal, and Hedrick (2001) and Xiao et al. (2009) studied the effect of time delays for leader following schemes under restrictions on controller structure.

Other researchers have studied more complex approaches to formation control that also present issues as the platoon size increases. In Jovanovic and Bamieh (2005), the authors study optimal control strategies for platoons with an increasing number of vehicles and show that some related LQR problems are "illposed". The work in Bamieh, Jovanovic, Mitra, and Patterson (2012) shows that in the 1-D case it is impossible to have large "coherent" platoons with only local feedback. A PDE approach was used by the authors of Barooah, Mehta, and Hespanha (2009) to show that the "stability margin" of a bidirectional control architecture can be improved by "mistuning". More recently Lin et al. (2012) integrated the previous results in the design of optimal controllers to enhance the coherence of a formation.

Leader following schemes can provide string stability and "tight formations" in which a fixed, prescribed inter-vehicle spacing is maintained, regardless of the platoon speed. These properties ensure some degree of safety and performance for the vehicles' manoeuvres and are important for applications where the tightness of the formations is required (e.g., increased throughput in Automated Highway Systems, Hedrick, Tomizuka, \& Varaiya, 1994).

The main contributions of this paper can be summarized as follows. We revisit the leader-predecessor following architecture where each follower tracks simultaneously the positions of its immediate predecessor and the leader. We also propose two novel alternatives based on it: the first one considers a modification of the way the leader position is communicated to the followers; the second one makes the followers track the velocity of the leader instead of its position. We provide formulae in the frequency domain for the dynamics of the resulting interconnections with and without the presence of time delays and for general linear controllers. Finally, we study the string stability properties of the three architectures mentioned above and their ability to achieve a tight formation.

The paper is organized as follows. Section 2 gives the notation and framework. Section 3 defines the control architectures to be studied and in Section 4 we present the corresponding vehicle dynamics. The main results of the paper are presented in Section 5.
Section 6 includes numerical examples that illustrate the results obtained and Section 7 gives some final remarks.

\section{Framework and problem formulation}

\subsection{Notation}

The notation used in this paper follows much of the standard systems and control literature. Lowercase is used for real scalar signals, $x: \mathbb{R} \rightarrow \mathbb{R}$ with specific values of the signal denoted by $x(t)$. Uppercase is used for scalar complex-valued Laplace transforms of signals and transfer functions, $X: \mathbb{C} \rightarrow \mathbb{C}$ with specific values denoted by $X(s)$. For the sake of brevity in the notation, where there is no confusion, the argument $(s)$ will be omitted. Vectors will be denoted as $\underline{x}(t) \in \mathbb{R}^{n}$ and $\underline{X} \in \mathbb{C}^{n}$, while $\underline{x}(t)^{\top}$ and $\underline{X}^{\top}$ denote their transposes. The imaginary unit is denoted by $j$, with $j^{2}=-1$. Boldface will be used for matrices $\boldsymbol{G} \in$ $\mathbb{C}^{n \times m}$ and the $(i, k)$ th entry of $\boldsymbol{G}$ is denoted by $G_{i, k}$. The magnitude of $X$ when $s=j \omega, \omega \in \mathbb{R}$, is denoted by $|X|$ and its magnitude peak over all possible values of $\omega$ is denoted as $\|X\|_{\infty}:=\sup _{\omega}|X(j \omega)|$. For $z \in \mathbb{C}, \Re(z)$ and $\Im(z)$ denote the real and imaginary parts of $z$ respectively.

\subsection{Vehicle model and preliminaries}

We consider a platoon of $n \in \mathbb{N}$ identical vehicles that travel in a straight line, with the aim of maintaining a desired and constant inter-vehicle spacing $\Delta>0$. The vehicle dynamics considered are linear and time invariant, namely

$m \ddot{x}_{i}(t)=-k_{d} \dot{x}_{i}(t)+d_{i}(t)+f\left(u_{i}(t)\right)$ for $1 \leq i \leq n$,

where: $x_{i}(t)$ denotes the position of the $i$ th vehicle along the string; $m$ its mass; $k_{d}$ is the vehicle drag coefficient; $f$ is the force applied by the engine, which is a function of $u_{i}(t)$, the control signal; and $d_{i}(t)$ is a disturbance force that acts on the vehicle. Assuming simple dynamics for the engine, i.e. $f\left(u_{i}(t)\right)=u_{i}(t)$, we can work in the frequency domain. For simplicity we assume that every car starts from rest and is initially positioned in the desired formation, that is $x_{i}(0)=(1-i) \Delta$ and $\dot{x}_{i}(0)=0$ for $i=1, \ldots, n$ and we define $\tilde{x}_{i}(t)=x_{i}(t)+(i-1) \Delta$. Therefore, taking the Laplace transform we obtain the frequency domain vehicle models

$\tilde{X}_{i}=\frac{U_{i}+D_{i}}{s\left(m s+k_{d}\right)}=X_{i}+(i-1) \frac{\Delta}{s}$ for $1 \leq i \leq n$.

Now, the control goal is to keep a tight formation, that is, to maintain the errors $e_{i}^{p r e}(t)=x_{i-1}(t)-x_{i}(t)-\Delta=\tilde{x}_{i-1}(t)-\tilde{x}_{i}(t)$ as close to zero as possible. This small error performance should be achieved in steady state, under disturbances to any member of the platoon, and for a constant speed of the leader. To achieve this, the control signal for each vehicle $u_{i}(t)$ is computed using the local measurement of the immediate predecessor position (indirectly through the measure of the inter-vehicle spacing) and the information being received from the leader (see Fig. 1). As a consequence, the leader-follower errors $e_{i}^{\text {lea }}(t)=x_{1}(t)-x_{i}(t)-$ $(i-1) \Delta=\tilde{x}_{1}(t)-\tilde{x}_{i}(t)$ will have a steady state response similar to that of $e_{i}^{\text {pre }}(t)$. These error signals can be associated with the performance of the system when considering traffic density issues and throughput. With this, we have that the Laplace transforms for the errors are given by

$E_{i}^{p r e}=X_{i-1}-X_{i}-\frac{\Delta}{s}=\tilde{X}_{i-1}-\tilde{X}_{i}$,

$E_{i}^{\text {lea }}=X_{1}-X_{i}-(i-1) \frac{\Delta}{s}=\tilde{X}_{1}-\tilde{X}_{i}$

These two errors will be central to our later analysis. 


\section{Architectures for formation control}

We consider three main approaches to achieve the platoon control objectives.

\subsection{Leader-predecessor following}

The leader-predecessor following structure is implemented by the use of the vehicle inputs

$U_{i}=K\left(\eta E_{i}^{p r e}+(1-\eta) E_{i}^{\text {lea }}\right)=K\left(\eta \tilde{X}_{i-1}+(1-\eta) \tilde{X}_{1}-\tilde{X}_{i}\right)$,

where $\eta \in(0,1)$ and $K$ is a dynamic compensator (in the present case taken to be identical for all vehicles) that stabilizes each vehicle in closed loop. Each car takes the weighted average spacing error of its predecessor and leader to regulate its own position. Some drawbacks of the scheme are: (i) each follower must be aware of its own position within the string since the leader-follower errors are given by $e_{i}^{\text {lea }}(t)=x_{1}(t)-x_{i}(t)-(i-1) \Delta$; (ii) each vehicle must have a highly accurate absolute position reference to compute $x_{1}(t)-x_{i}(t)$; (iii) each follower must use the same value for $\Delta$, imposing a coordination requirement. These can be overcome by the schemes described below.

\subsection{Leader velocity tracking and predecessor following}

Here we drop the leader position knowledge for each vehicle, exchanging it for measurements of the position of its predecessor and the velocity of the leader. The control signal for each car takes the form

$U_{i}=K_{p} E_{i}^{p r e}+K_{v} s E_{i}^{\text {lea }}=\tilde{K} \frac{K_{p}}{\tilde{K}} \tilde{X}_{i-1}+1-\frac{K_{p}}{\tilde{K}} \quad \tilde{X}_{1}-\tilde{X}_{i}$,

where $K_{p}, K_{v}$ and $\tilde{K}=K_{p}+s K_{v}$ are dynamic compensators such that $\tilde{K}$ stabilizes each vehicle in closed loop. This choice aims to keep a tight inter-vehicle spacing and simultaneously track the leader velocity in every follower. One advantage of this architecture is that each follower does not need to be aware of its position along the string. New members can join the rear of the platoon without having to know the number of vehicles that separate them from the leader. Every follower has to listen to $\dot{x}_{1}(t)$ (see Fig. 1) while measuring with local sensors (e.g. radar) its distance to its predecessor.

Remark 1. This scheme has a connection with time headway policies. This can be seen from (6), which can be written as:

$U_{i}=K_{p} \quad \tilde{X}_{i-1}-\tilde{X}_{i}-\frac{K_{v}}{K_{p}} s \tilde{X}_{i}+K_{v} s \tilde{X}_{1}$.

Therefore, in terms of the local dynamics, the feedback includes the equivalent of a (possibly dynamic) time headway, $K_{v} / K_{p}$. The use of the leader velocity means that improved dynamic properties may be achieved without increasing the steady state vehicle separation that commonly occurs with time headway policies (Chien \& Ioannou, 1992).

\subsection{Alternative algorithm: indirect leader state broadcast}

Leader state information need not be sent directly to each member. Alternatively it can be computed indirectly at any follower from local measurements and information being sent by its predecessor. For instance, consider the control signal

$U_{i}=K\left(E_{i}^{p r e}+(1-\eta) \Gamma \mathrm{E}_{i-1}\right)$,

where $\mathrm{E}_{i-1}$ is a signal sent by the $(i-1)$ th member, containing an estimate of the state of the leader and $\Gamma$ is a stable transfer function. In the particular case $\mathrm{E}_{i-1}=E_{i-1}^{\text {lea }}$ and $\Gamma=1$, i.e. the estimation is perfect, the control signals coincide with the first case of leader-predecessor following

$U_{i}=K\left(E_{i}^{p r e}+(1-\eta) E_{i-1}^{\text {lea }}\right)=K\left(\eta \tilde{X}_{i-1}+(1-\eta) \tilde{X}_{1}-\tilde{X}_{i}\right)$,

and the resulting dynamics are identical to (5).

In the general case, each member receives $\Gamma \mathrm{E}_{i_{-1}}$ from its predecessor assuming that it is exactly $E_{i-1}^{\text {lea }}$. This allows any vehicle to estimate their own leader-follower error adding the local measurement $E_{i}^{\text {pre }}$ to the received signal, that is

$\mathrm{E}_{i}=\Gamma \mathrm{E}_{i-1}+E_{i}^{p r e}$,

which in turn is sent to the immediate follower. The main benefit of this alternative is that the coordination requirements of the leader-predecessor following are no longer needed.

\section{Closed loop dynamics}

In this section, we derive formulae that will allow us to study the properties of the three architectures defined in Section 3. In particular we are interested in studying the effect of time delays in the reception of the leader state and the following derivations prove to be useful in that regard.

\subsection{Vehicle string dynamics for direct leader state broadcast schemes}

The two unidirectional control structures introduced in Sections 3.1 and 3.2 share a similar mathematical description, differing only in the compensator and the weights of the errors. Moreover, they belong to a broader class of MIMO systems. In particular we consider the class of control laws

$U_{i}=K\left(P \tilde{X}_{i-1}+L_{i} \tilde{X}_{1}-\tilde{X}_{i}\right)$,

where $L_{i}$ and $P$ are arbitrary stable transfer functions such that $L_{i}(0)+P(0)=1$. The constraint on the DC gains of $L_{i}$ and $P$ is required to ensure bounded control signals $u_{i}(t)$ when the vehicles move at a constant speed (and for consistency with (5) and (6)). Here we allow the leader transfer function $L_{i}$ to vary, while considering homogeneous predecessor transfer functions $P$. This will allow us to include a factor that accounts for time delays in the reception.

Remark 2. In some of the following discussions when referring to the leader-predecessor following architecture we will note it by stating $P=\eta \in(0,1)$. Otherwise, a dynamic $P$ will be referring to leader velocity tracking.

We are interested in the response of the string to input disturbances on the vehicles. First, we define the vehicle model transfer function as

$\tilde{H}=\frac{1}{m s+k_{d}} \Rightarrow H=\frac{\tilde{H}}{s}$,

and we let

$T=H K(1+H K)^{-1}, \quad S=1-T$

be the usual closed loop sensitivity functions. Then, if the lead vehicle does not possess a controller and it drives independently, the vehicle dynamics are given by

$\underline{\tilde{X}}=(\boldsymbol{I}-H K \boldsymbol{G})^{-1} H \underline{D}$,

where $\underline{\tilde{X}}=\tilde{X}_{1} \cdots \tilde{X}_{n}{ }^{\top}, \boldsymbol{I}$ is the $n \times n$ identity matrix, $\underline{D}=$ $\left[D_{1} \cdots D_{n}\right]^{\top}$ and $\boldsymbol{G} \in \mathbb{C}^{n \times n}$ is the interconnection matrix:

$\boldsymbol{G}=\begin{array}{ll}0 & \underline{0}^{\top} \\ \underline{L} & \end{array}$ 
where $\underline{L}=\left[\begin{array}{lll}L_{2} L_{3} & \cdots & L_{n}\end{array}\right]^{\top}, \underline{0} \in \mathbb{R}^{(n-1)}$ is the zero vector and the matrix $8 \in \mathbb{C}^{(n-1) \times(n-1)}$ is given by $8_{i, i}=-1$ for $1 \leq i \leq n-1$, $8_{i+1, i}=P$ for $1 \leq i \leq n-2$ and all other entries of 8 are zero. Note that, the selection $G_{1,1}=0$ represents the freedom of movement for the lead vehicle. In other words, its trajectory will be determined only by $X_{1}=H D_{1}$. Corresponding results to those presented here can be derived for alternative formulations using a fictitious leader or other strategies, but the essential results that follow remain unaltered.

Now, the matrix $(I-H K G)$ and its inverse have the following structures

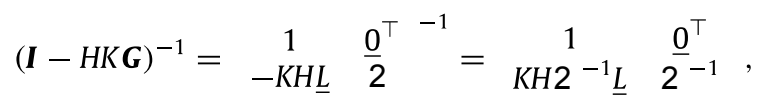

where $2 \in \mathbb{C}^{(n-1) \times(n-1)}$ satisfies $\Theta_{i, i}=K H T^{-1}$ for $1 \leq i \leq n-1$, $\Theta_{i+1, i}=-K H P$ for $1 \leq i \leq n-2$ and all other entries of 2 are zero.

Computing $2^{-1}$ is straightforward since 2 is lower triangular

$$
2^{-1}=\frac{T}{K H} \quad \begin{array}{ccccc}
1 & & & \\
& \vdots & \ddots & & \\
& (P T)^{n-2} & \ldots & P T & 1
\end{array}
$$

Under these considerations, disturbances at any member of the string will affect the vector of predecessor errors $\underline{E}^{\text {pre }}=E_{2}^{\text {pre }} \cdots E_{n}^{\text {pre }}{ }^{\top}$ as

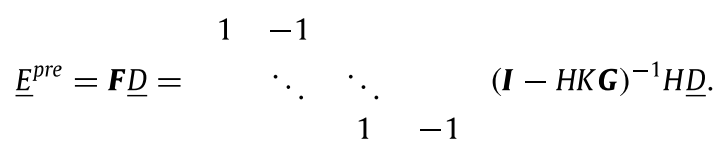

\subsection{Vehicle string dynamics for indirect leader state broadcast}

The string dynamics for the alternative algorithm from Section 3.3 can be determined by first inserting (8) in (2) and taking the difference in position for two successive followers

$$
\begin{aligned}
\tilde{X}_{i-1}= & H K\left((1-\eta) \Gamma \mathrm{E}_{i-2}+E_{i-1}^{p r e}\right)+H D_{i-1}, \\
\tilde{X}_{i}=H K & \left((1-\eta) \Gamma \mathrm{E}_{i-1}+E_{i}^{\text {pre }}\right)+H D_{i}, \\
E_{i}^{\text {pre }}= & H K\left((1-\eta)(1-\Gamma) \mathrm{E}_{i-1}+\eta E_{i-1}^{\text {pre }}-E_{i}^{\text {pre }}\right) \\
& \quad+H\left(D_{i-1}-D_{i}\right) .
\end{aligned}
$$

Merging the last equation with (10) we can write

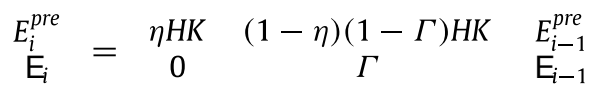

$$
\begin{aligned}
& \times \begin{array}{ccc}
H K & 0 & E_{i}^{p r e} \\
-1 & 0 & \mathrm{E}_{i}
\end{array}+H \begin{array}{c}
D_{i-1}-D_{i} \\
0
\end{array},
\end{aligned}
$$

for $i \geq 3$.

For this case we will only focus on disturbances at the leader and set $D_{i}=0$ for $i=2, \ldots, n$. For the first follower, i.e. $i=2$, we have

$$
\begin{gathered}
E_{2}^{\text {pre }} \\
\mathrm{E}_{2}
\end{gathered}=-\begin{array}{ccc}
H K & 0 & E_{2}^{p r e} \\
-1 & 0 & \mathrm{E}_{2}
\end{array}+H \begin{gathered}
D_{1} \\
0
\end{gathered}
$$

and hence, $E_{2}=E_{2}^{p r e}=S H D_{1}$. With these facts we can solve the first order recursion in (22) obtaining the desired dynamics

$$
\begin{gathered}
E_{i}^{p r e} \\
\mathrm{E}_{i}
\end{gathered}=\begin{array}{lccc}
\eta T & (1-\eta)(1-\Gamma) T & { }^{i-2} & 1 \\
\eta T & (1-\eta)(1-\Gamma) T+\Gamma & 1
\end{array} S D_{1} .
$$

\section{Properties of the interconnections}

In this section, we present the main results of this paper. Our approach to string stability is to show that certain transfer functions from disturbances to errors have a magnitude peak with a bound that is independent of the string length (Middleton \& Braslavsky, 2010). We use the following definition.

Definition 1. Let $\left\{F_{n}\right\}$ be a sequence of stable transfer functions. The sequence will be called string stable if there exists $c \in \mathbb{R}$, independent of $n \in \mathbb{N}$ such that $\left\|F_{n}\right\|_{\infty} \leq c$ for all $n$. It will be called string unstable otherwise.

Remark 3. In the following we assume that $K$ has at least one pole at $s=0$, which is a requirement for the string to achieve zero velocity tracking error for constant velocity trajectories of the leader.

The following lemma, stated in Seiler et al. (2004) and Middleton (1991) implies string instability under the assumptions given above in the leader-predecessor following scheme when there is no leader communication.

Lemma 1. Let $T$ be a real rational scalar function of the complex variable s. Suppose that $T(0)=1$ and also that $T$ is stable (analytic in the closed right half complex plane). Then

$$
{ }_{0}^{\infty} \ln |T(j \omega)| \frac{d \omega}{\omega^{2}} \geq \frac{\pi}{2} T^{\prime}(0)
$$

In the current case, $T$ is given by (13). Since the dynamics considered for vehicles and controller are such that the term $H K$ has two poles at the origin, it is easy to see that $T^{\prime}(0)=0$. Therefore, Lemma 1 implies that $\|T\|_{\infty}>1$.

In the leader-predecessor following scheme, it is straightforward from (5) that with no leader communication, i.e. $\eta=1$, the sequence of predecessor errors for a disturbance $D_{1}$ at the leader will be given by $E_{n}^{\text {pre }}=H S T^{n-2} D_{1}=F_{n} D_{1}$. Since $H$ and $K$ have a finite number of zeros it is possible to choose $\omega_{0}$ such that $\left|H\left(j \omega_{0}\right) S\left(j \omega_{0}\right)\right| \neq 0$ and $\left|T\left(j \omega_{0}\right)\right|>1$ (Seiler et al., 2004). Therefore, we have that the sequence $\left\{F_{n}\right\}=\left\{H S T^{n-2}\right\}$ is string unstable according to Definition 1 . The following simple proposition will help in the presentation of the main results.

Proposition 1. Let PT be a stable and proper transfer function such that $\|P T\|_{\infty} \leq 1$. Also let $W, Q$ be transfer functions such that $\|W\|_{\infty},\|Q\|_{\infty}$ are well defined and $W+Q(P T)^{n}$ is stable for every $n \in \mathbb{N}$. Then, the sequence $\left\{W+Q(P T)^{n}\right\}$ is string stable.

Proof. First, we have from the triangle inequality that $\mid W+$ $Q(P T)^{n}|\leq| W|+| Q \|(P T)^{n} \mid$ holds for all $\omega \geq 0, n \in \mathbb{N}$. Since $\|P T\|_{\infty} \leq 1$ it is also true that $|W|+|Q|\left|(P T)^{n}\right| \leq|W|+|Q|$. If we let $\|W\|_{\infty}=a$ and $\|Q\|_{\infty}=b$ then $\left|W+Q(P T)^{n}\right| \leq a+b$ for all $\omega \geq 0, n \in \mathbb{N}$. Hence, the sequence $\left\{W+Q(P T)^{n}\right\}$ is string stable according to Definition 1 with $c=a+b \in \mathbb{R}$.

\subsection{Disturbances at followers: direct leader state broadcast schemes}

A disturbance $D_{k}$ at the $k$ th follower, where $k$ is fixed, will affect the $n$th predecessor error $E_{n}^{p r e}, n \geq k>1$, through the entry $F_{n, k}$ of the matrix $\boldsymbol{F}$ defined in (18). In other words

$E_{n}^{\text {pre }}=F_{n, k} D_{k}$,

with

$F_{n, k}=\begin{array}{ll}(1-P T)(P T)^{n-k-1} S H & \text { if } n>k \\ S H & \text { if } n=k .\end{array}$ 
If $\mathrm{F}_{n, k}={ }_{i=k}^{n} F_{n, k}$, the corresponding leader errors are given by

$E_{n}^{\text {lea }}={ }_{i=k}^{n} E_{i}^{\text {pre }}=\mathrm{F}_{n, k} D_{k}=\left(2-(P T)^{n-k}\right) S H D_{k}$,

that is, $\mathrm{F}_{n, k}$ is the transfer function from a disturbance at the $k$ th member to the $n$th leader error.

The selection of $L_{i}$ (which determines the leader information reception) does not affect these responses directly. Moreover, disturbances at the followers will not affect predecessors (which is obvious from the interconnection).

Theorem 1 (Disturbances at the Followers). Consider the sequences $\left\{F_{n, k}\right\}$ and $\left\{F_{n, k}\right\}$ given by (27) and (28) respectively. The following statements are true:

(1) $F_{n, k}(0)=\mathrm{F}_{n, k}(0)=0$ for all $n \geq k>1$.

(2) If $\|P T\|_{\infty} \leq 1$, then $\left\{F_{n, k}\right\}$ and $\left\{\bar{F}_{n, k}\right\}$ are string stable.

Proof. (1) Since the product $K H$ has exactly two poles at $s=0$, we can write $S=s^{2} \tilde{S}$ with $\tilde{S}(0) \neq 0(S$ has two zeros at $s=0)$. The vehicle transfer function is $H=\tilde{H} / s, \tilde{H}(0) \neq 0$ as defined in (18). From their definitions $P$ and $T$ are stable and consequently we can rewrite $F_{n, k}=s(1-P T)(P T)^{n-k-1} \tilde{S} \tilde{H}$. Evaluating at $s=0$ yields $F_{n, k}(0)=0$ for $n \geq k>1$. From its definition $\mathrm{F}_{n, k}={ }_{i=k}^{n} F_{n, k}$ and therefore $\mathrm{F}_{n, k}(0)={ }_{i=k}^{n} F_{n, k}(0)=0$, for $n \geq k>1$ which is the required result.

(2) For $\left\{F_{n, k}\right\}$ Proposition 1 holds with $W=0$ and $Q=(1-$ $P T) S H$. For $\left\{\mathrm{F}_{n, k}\right\}$ it holds with $W=2 S H Q=-S H$.

The first part of Theorem 1 implies that constant disturbances at the followers will yield zero steady state errors. String stability of $\left\{F_{n, k}\right\}$ implies that disturbances at the $k$ th member are not amplified for the remaining followers $(n>k)$, accounting for a string safety condition. Moreover, string stability of $\left\{\mathrm{F}_{n, k}\right\}$ ensures that disturbances at the $k$ th follower do not create increasing leader-follower spacings.

On the other hand, suppose there exists $\omega_{0} \geq 0$ for which $P\left(j \omega_{0}\right) T\left(j \omega_{0}\right)=\gamma e^{j \theta}$ with $\gamma>1$. Since the product $S H$ has no poles at the origin (the pole of $H$ is cancelled by one of the zeros of $S$ ) and is stable, we have $\left|S\left(j \omega_{0}\right) H\left(j \omega_{0}\right)\right|=\alpha<\infty$. Moreover, $P T$ is stable which implies $\left|1-P\left(j \omega_{0}\right) T\left(j \omega_{0}\right)\right|=\beta<\infty$ and therefore

$\left|F_{n, k}\left(j \omega_{0}\right)\right|=\alpha \beta \gamma^{n-k+1}$,

$\left|\mathrm{F}_{n, k}\left(j \omega_{0}\right)\right|=\alpha\left|2-\gamma^{n-k+1} e^{j(n-k+1) \theta}\right|$,

for all $n \geq k>1$. Therefore, in this case the disturbance amplification will grow unbounded with the string length, i.e. we will have string instability in (27).

Remark 4. Our assumptions on the vehicle and controller dynamics $H$ and $K$ imply that $\|T\|_{\infty}>1$. Therefore the design of $P$ through the leader state communication must be aimed to achieve at least $\|P T\|_{\infty} \leq 1$. For the leader-following approach $P=\eta \in(0,1)$ and it suffices to have $\eta \leq\|T\|_{\infty}^{-1}$. However, for the leader velocity tracking approach $K=K_{p}+s K_{v}$ and $P=K_{p} /\left(K_{p}+s K_{v}\right)$ where $K_{p}$ and $K_{v}$ are stable transfer functions. This means that

$P T=\frac{K_{p} H}{1+H\left(K_{p}+s K_{v}\right)}=K_{p} H S$,

and $K_{p}, K_{v}$ must be designed in order to satisfy at least $\|P T\|_{\infty} \leq 1$. Whether it is possible to satisfy this condition for any $H$ with marginally stable (for integration) $K_{p}$ and $K_{v}$ is the subject of ongoing research (although it can be seen that $K=K_{p}+s K_{v}$ can be fixed to achieve closed loop stability and $K_{p}$ can be designed to achieve the bound, the question is if the resulting $K_{v}=\left(K-K_{p}\right) / s$ remains stable). In the following we will be interested in transfer functions $P$ such that $P(0)=1$ and $\|P T\|_{\infty} \leq 1$. We will provide some numerical examples that show the feasibility of satisfying this condition.

5.2. Disturbances at the lead vehicle: direct leader state broadcast schemes

Now we focus our attention on the effect of disturbances to the lead vehicle. From (18), the inter-vehicle spacing transfer function for the last member of the string when a disturbance in the leader occurs is given by

$F_{n, 1}=(1-P T){ }_{i=2}^{n-1} L_{i}(P T)^{n-i-1}-L_{n} \quad T H$.

The transfer function that describes the effect of $D_{1}$ on the leader error for the last member, that is $E_{n}^{\text {lea }}$, is given by

$\mathrm{F}_{n, 1}={ }_{i=2}^{n} F_{i, 1}$

In general $L_{2}=1$ since the second member of the platoon just follows the leader using its predecessor error $E_{2}^{\text {pre }}=\tilde{X}_{1}-\tilde{X}_{2}$. The elements $L_{i}$ for $i \geq 3$ can be used to describe the reception of the leader information along the string. We are mainly interested in the effect of time delays on the resulting DC gains and string stability conditions for the transfer functions mentioned above. Three cases are studied.

\section{(a) Perfect communication}

The case of perfect reception of the leader information can be studied when $L_{i}=1-P$ for $i=3, \ldots, n$. In this case (32) and (33) yield

$F_{n, 1}=S H(P T)^{n-2}$

$\mathrm{F}_{n, 1}=S H{ }_{i=2}^{n}(P T)^{i-2}=S H \frac{1-(P T)^{n-1}}{1-P T}$.

We have the following result.

Theorem 2 (Disturbances at the Leader: Perfect Communication). Consider the sequences $\left\{F_{n, k}\right\}$ and $\left\{F_{n, k}\right\}$ given by (34) and (35) respectively. The following statements are true:

(1) $F_{n, k}(0)=F_{n, k}(0)=0$;

(2) if $\|P T\|_{\infty} \leq 1,\left\{F_{n, 1}\right\}$ is string stable;

(3) if $\|P T\|_{\infty} \leq 1$ and $P T \neq 1$ for $s=j \omega, \omega>0$, then $\left\{F_{n, 1}\right\}$ is string stable.

Proof. (1) The proof is analogous to part (1) of Theorem 1.

(2) Proposition 1 holds with $W=0$ and $Q=S H$, which implies string stability of the sequence $\left\{F_{n, 1}\right\}$.

(3) If $\|P T\| \leq 1, P T \neq 1$ for $s=j \omega, \omega>0$, then the magnitudes $|W|=|S H /(1-P T)|$ and $|Q|=|S H /(P T-1)|$ are well defined for $\omega>0$. If $P=\eta \in(0,1)$ or $P(0) \neq 1$, evaluating yields $W(0)=Q(0)=0$. For dynamic $P$ such that $P(0)=1$ (and therefore $P(0) T(0)=1$ ) it is easy to compute

$\lim _{s \rightarrow 0} \frac{S H}{1-P T}=\lim _{s \rightarrow 0} \frac{s \tilde{S} \tilde{H}}{1-P T}=-\frac{\tilde{S}(0) \tilde{H}(0)}{P^{\prime}(0)}$,

which implies that $\|W\|_{\infty}$ and $\|Q\|_{\infty}$ are well defined for any $P$ satisfying the conditions. Hence, Proposition 1 shows that the sequence $\left\{\mathrm{F}_{n, 1}\right\}$ is string stable.

If there is perfect reception of the leader state, and disturbances occur at the lead vehicle, Theorem 2 shows that the 
two architectures with direct leader state broadcast, namely leader-predecessor following and leader velocity tracking, achieve a tight formation. Also, both architectures are string stable for the error signals of interest, for appropriately designed $P$, differing only in the resulting dynamics.

It can be noted that there is an extra condition for the string stability of $\left\{\mathrm{F}_{n, 1}\right\}$, when compared to Theorem 1 . Let $P T=e^{j \theta}$ at some $\omega=\omega_{0}$, then, if $\theta=2 k \pi k \in \mathbb{Z}$, that is $P T=1$ at $s=j \omega_{0}$, we have

$$
\lim _{\omega \rightarrow \omega_{c}} \frac{1-(P T)^{n-1}}{1-P T}=\lim _{\theta \rightarrow 2 k \pi} \frac{1-e^{j \theta(n-1)}}{1-e^{j \theta}}=n-1,
$$

and consequently, $\left|F_{n, 1}\left(j \omega_{0}\right)\right|=\left|S\left(j \omega_{0}\right) H\left(j \omega_{0}\right)\right|(n-1)$. This means that the disturbance amplification grows linearly with the size of the platoon and we have string instability.

\section{(b) One-step string relay communications}

Here we study the effects of a single occurrence of time delay on the architectures. Choose some $n_{r}$ with $3 \leq n_{r}<n$ and setting $L_{i}=1-P$ for $i=3, \ldots, n_{r}$ and $L_{i}=(1-P) e^{-\tau s}, \tau>0$ for $i=n_{r}+1, \ldots, n$. This corresponds to one rebroadcast of the leader state with a delay $\tau$, by the $n_{r}$ th member of the string. For this case (32), when $n>n_{r}$, is given by

$$
\begin{aligned}
F_{n, 1}= & T H(1-P T)(P T)^{n-3}+(1-P T)(1-P)_{i=3}^{n_{r}}(P T)^{n-i-1} \\
& +(1-P T)(1-P) e^{-\tau s}{ }_{i=n_{r}+1}^{n-1}(P T)^{n-1-i}-L_{n} \\
= & T H(P T)^{n-3} P S+\left(1-e^{-\tau s}\right)(1-P)(P T)^{n-n_{r}-1} .
\end{aligned}
$$

To compute (33) first we note that

$$
\mathrm{F}_{n, 1}={ }_{i=2}^{n} F_{i, 1}={ }_{i=2}^{n_{r}} F_{i, 1}+{ }_{i=n_{r}+1}^{n} F_{i, 1} \text {, }
$$

where we separate terms for vehicles without time delay, from those with delay. Inserting the formulae obtained above for $F_{i, 1}$ with and without delay we obtain

$\mathrm{F}_{n, 1}=S H \frac{1-(P T)^{n-1}}{1-P T}+\left(1-e^{-\tau s}\right)(1-P) T H \frac{1-(P T)^{n-n_{r}}}{1-P T}$.

Substituting $\tau=0$ yields the same expressions as in the perfect communication case.

Theorem 3 (Disturbances at the Leader: One-Step String Relay Communication). Consider the sequences $\left\{F_{n, k}\right\}$ and $\left\{\mathrm{F}_{n, k}\right\}$ given by (38) and (40) respectively. If $\tau>0,3 \leq n_{r}<n$, then the following holds:

(1) if $P=\eta \in(0,1)$ then $F_{n, 1}(0)=\tau \tilde{H}(0)(1-\eta) \eta^{n-n_{r}-1}$ and $F_{n, 1}(0)=\tau \tilde{H}(0)\left(1-\eta^{n-n_{r}}\right)$. If $P$ is dynamic with $P(0)=1$ then $F_{n, 1}(0)=F_{n, 1}(0)=0$;

(2) if $\|P T\|_{\infty} \leq 1,\left\{F_{n, 1}\right\}$ is string stable;

(3) if $\|P T\|_{\infty} \leq 1$ and $P T \neq 1$ for $s=j \omega, \omega>0$, then $\left\{F_{n, 1}\right\}$ is string stable.

Proof. See Appendix A.

The previous result states that both architectures are affected in different ways if there is a time delay in the reception of the leader state-in particular, time delays after a certain position along the string. We see that the tight formation is lost when $\tau>0$ for the leader-predecessor following scheme, whereas leader velocity tracking still provides 0 DC gain on both transfer function errors $F_{n, 1}$ and $F_{n, 1}$. Both architectures remain string stable for both sequences $\left\{F_{n, 1}\right\}$ and $\left\{F_{n, 1}\right\}$, when the leader state reception is delayed $\tau$ seconds for vehicles behind the $n_{r}$ th member.

\section{(c) Multi-step string relay communications}

An increasing time delay along the string can be seen as a worst case of communication constraints where multiple re-broadcasting along the string is required. This corresponds to the selections $L_{i}=(1-P) e^{-(i-2) \tau s}$ for $i=3, \ldots, n$. Now, the predecessor error transfer function is given as

$$
\begin{aligned}
F_{n, 1}= & T H(1-P T)(P T)^{n-3}-(1-P) e^{-(n-2) \tau s} \\
& +(1-P T)(1-P){ }_{i=3}^{n-1} e^{-(i-2) \tau s}(P T)^{n-1-i}
\end{aligned}
$$

With some more manipulations we obtain

$$
\begin{aligned}
F_{n, 1}= & T H(1-P T)(P T)^{n-3}-(1-P) e^{-(n-2) \tau s} \\
& +(1-P T)(1-P) e^{-\tau s} \frac{\left((P T)^{n-3}-e^{-(n-3) \tau s}\right)}{P T-e^{-\tau s}} .
\end{aligned}
$$

Proceeding in a similar fashion as in the previous case, we can write the leader error transfer function as

$$
\begin{aligned}
\mathrm{F}_{n, 1}= & H \frac{\left(1-(P T)^{n-1}\right)\left(e^{-\tau s}-T\right)}{e^{-\tau s}-P T} \\
& +\frac{(1-P)\left(1-e^{-(n-1) \tau s}\right) T}{e^{-\tau s}-P T} .
\end{aligned}
$$

Once more, setting $\tau=0$ results in the expression for the perfect communications case.

Theorem 4 (Disturbances at the Leader: Multi-Step String Relay Communication). Consider the sequences $\left\{F_{n, k}\right\}$ and $\left\{F_{n, k}\right\}$ with elements defined in (42) and (43) respectively. If $\tau>0$, then the following holds:

(1) if $P=\eta \in(0,1)$ then

$F_{n, 1}(0)=\tau \tilde{H}(0)\left(1-\eta^{n-2}\right)$,

$\mathrm{F}_{n, 1}(0)=\tau \tilde{H}(0) \quad n-1-\frac{1-\eta^{n-1}}{1-\eta}$.

If $P$ is dynamic with $P(0)=1$ then $F_{n, 1}(0)=F_{n, 1}(0)=0$;

(2) let $\|P T\|_{\infty} \leq 1$, and $P T-e^{-\tau s} \neq 0$ for $s=j \omega, \omega>0$. If $\tau \neq-P^{\prime}(0)$ the sequence $\left\{F_{n, 1}\right\}$ is string stable;

(3) the sequence $\left\{F_{n, 1}\right\}$ is string unstable for any selection of $P$.

Proof. See Appendix B.

If the amount of time delay for the reception of the leader state increases at every member, Theorem 4 states that neither architecture can provide string stability for $\left\{\mathrm{F}_{n, 1}\right\}$. This means that the leader error $E_{n}^{\text {lea }}=\tilde{X}_{1}-\tilde{X}_{n}$ will grow unbounded with the platoon size, implying a degradation in the performance of the string. On the other hand, both architectures achieve string stability on the predecessor errors, provided that $P$ is designed properly. This ensures a degree of safety, even when the time delay increases progressively along the string. The key difference between the two architectures is the values of the $D C$ gains $F_{n, 1}(0)$ and $F_{n, 1}(0)$. When the platoon travels at a constant speed the leader velocity tracking scheme provides a tight formation under the communication constraint, whereas the leader-predecessor following scheme will 
have spacings that differ from the desired formation with an offset that increases with the amount of time delay as seen from (44) and (45).

Remark 5. Part (2) of Theorem 4 shows that a condition for string stability is that $\tau \neq-P^{\prime}(0)$, which is only relevant for dynamic $P$, that is, for the leader velocity tracking case. This critical value for the time delay yields string instability in the predecessor errors (see Appendix B for details). However, for time delays larger or smaller than this critical value, which depends on the design of $P$. the leader velocity tracking architecture remains string stable in $\left\{F_{n, 1}\right\}$.

5.3. Disturbances at lead vehicle: indirect leader state broadcast schemes

We study the string stability properties of the dynamics obtained from the use of the alternative algorithm defined in (8). Only time delay on the communications will be studied, that is $\Gamma=e^{-\tau s}$.

The $n$th predecessor error is affected by a disturbance on the leader as $E_{n}^{\text {pre }}=F_{n, 1} D_{1}$ which can be computed from (24) by expanding the powers of the matrix

$$
\begin{array}{lcccc}
\eta T & (1-\eta)\left(1-e^{-\tau s}\right) T \\
\eta T & (1-\eta)\left(1-e^{-\tau s}\right) T+e^{-\tau s}=e^{-\tau s} \mathrm{M}
\end{array}
$$

We will prove that for any selection of the parameters $H, K$ or $\eta$, the existence of time delay in the estimation of the leader error, i.e. $\Gamma=e^{-\tau s}$, with $\tau>0$ will yield string instability. The following lemma will aid us in that regard.

Lemma 2. Let $T$ be any stable rational transfer function, and consider the real parameters $\eta \in\left(0,\|T\|_{\infty}^{-1}\right]$ and $\tau>0$. Then, the spectral radius of the matrix

$\mathrm{M}(s)=\begin{array}{cc}\eta T & (1-\eta)\left(e^{\tau s}-1\right) T \\ \eta T & (1-\eta)\left(e^{\tau s}-1\right) T+1\end{array}$,

satisfies $|\rho(\mathrm{M}(j \omega))|>1$ at some $\omega \in \mathbb{R}$.

Proof. If $\tau>0$, we note that for values $s=j \omega_{k}$ with $\omega_{k}=$ $2 k \pi / \tau, k \in \mathbb{Z}$, the factor $e^{\tau s}-1$ vanishes, M $\left(j \omega_{k}\right)$ becomes lower triangular and therefore the eigenvalues satisfy $\lambda_{1}\left(j \omega_{k}\right)=\eta T\left(j \omega_{k}\right)$ and $\lambda_{2}\left(j \omega_{k}\right)=1$ at these frequencies. Since the equality $\eta T\left(j \omega_{k}\right)=$ 1 can only hold at a finite number of these $\omega_{k}$ (given that $T$ is real rational), we have that the eigenvalues $\lambda_{1}\left(j \omega_{k}\right), \lambda_{2}\left(j \omega_{k}\right)$ are algebraically simple for infinitely many $\omega_{k}$. Now, for algebraically simple eigenvalues, Theorem 6.3.12 of Horn and Johnson (1999) can be applied to $\mathrm{M}\left(j \omega_{k}\right)$ yielding the derivative of $\lambda_{2}(j \omega)$ with respect to $\omega$ at $\omega=\omega_{k}$ as

$\lambda_{2}^{\prime}\left(j \omega_{k}\right)=\frac{\underline{y}^{*} \mathrm{M}{ }^{\prime}\left(j \omega_{k}\right) \underline{x}}{y^{*} \underline{x}}$,

where $\underline{x}, y$ are the right and left eigenvectors of $\mathrm{M}\left(j \omega_{k}\right)$ corresponding to $\lambda_{2}\left(j \omega_{k}\right)$ respectively and $(\cdot)^{*}$ denotes the conjugate transpose. In particular, as

$\mathrm{M}\left(j \omega_{k}\right)=\begin{array}{ll}\eta T\left(j \omega_{k}\right) & 0 \\ \eta T\left(j \omega_{k}\right) & 1\end{array}$,

the right and left eigenvectors $\underline{x}, \underline{y}$ corresponding to $\lambda_{2}\left(j \omega_{k}\right)$ are given by

$\underline{x}=\begin{gathered}0 \\ 1\end{gathered}, \quad \underline{y}^{*}=\frac{\eta T\left(j \omega_{k}\right)}{1-\eta T\left(j \omega_{k}\right)} \quad 1$
The derivative $\mathrm{M}{ }^{\prime}\left(j \omega_{k}\right)$ takes the form

$\mathrm{M}^{\prime}\left(j \omega_{k}\right)=\begin{array}{ll}\eta T^{\prime}\left(j \omega_{k}\right) & j(1-\eta) \tau T\left(j \omega_{k}\right) \\ \eta T^{\prime}\left(j \omega_{k}\right) & j(1-\eta) \tau T\left(j \omega_{k}\right)\end{array}$,

and substituting these expressions in (48) results in

$\lambda_{2}^{\prime}\left(j \omega_{k}\right)=\frac{j(1-\eta) \tau T\left(j \omega_{k}\right)}{1-\eta T\left(j \omega_{k}\right)}$.

If we consider the derivative of $\left|\lambda_{2}(j \omega)\right|^{2}$ with respect to $\omega$ at $\omega=\omega_{k}$ we have

$\left(\lambda_{2}^{*}(j \omega) \lambda_{2}(j \omega)\right)^{\prime}{ }_{\omega=\omega_{k}}=\left(\lambda_{2}^{\prime}\left(j \omega_{k}\right)\right)^{*}+\lambda_{2}^{\prime}\left(j \omega_{k}\right)$,

since $\lambda_{2}\left(j \omega_{k}\right)=1$ and therefore $\left|\lambda_{2}(j \omega)\right|>1$ either at $\omega>\omega_{k}$ or $\omega<\omega_{k}$ if and only if $\Re \lambda_{2}^{\prime}\left(j \omega_{k}\right) \neq 0$. Now, from (48) $\Re \lambda_{2}^{\prime}\left(j \omega_{k}\right)=0$ if and only if $\Im\left\{T\left(j \omega_{k}\right) /\left(1-\eta T\left(j \omega_{k}\right)\right)\right\}=0$, however, recalling that $T$ is a rational function this can at most be true at a finite number of frequencies. Consequently, since there are infinitely many $\omega_{k}, \Im\left\{T\left(j \omega_{k}\right) /\left(1-\eta T\left(j \omega_{k}\right)\right)\right\} \neq 0$ and $\left|\lambda_{2}(j \omega)\right|>$ $\left|\lambda_{2}\left(\omega_{k}\right)\right|=1$ holds for some $\omega \neq \omega_{k}$.

Theorem 5. Let $E_{n}^{\text {pre }}=F_{n, 1} D_{1}$ where $F_{n, 1}$ is computed from (24). Then, if $\tau>0$ and $\|\eta T\|_{\infty} \leq 1$ the sequence $\left\{F_{n, 1}\right\}$ is string unstable.

Proof. Lemma 2 states that there exists $\omega_{c}$ such that the spectral radius of $\mathrm{M}\left(j \omega_{c}\right)$ satisfies $\rho\left(\mathrm{M}\left(j \omega_{c}\right)\right)>1$, then $\lambda_{1}\left(j \omega_{c}\right) \neq \lambda_{2}\left(j \omega_{c}\right)$ since $|\operatorname{det} \mathrm{M}(j \omega)|=|\eta T| \leq 1$ for all $\omega \geq 0$. Now, the following decomposition holds at all $\omega$ such that $\rho(\mathrm{M}(j \omega))>1$

$\mathrm{M}(j \omega)^{i-2} \quad \begin{aligned} & 1 \\ & 1\end{aligned}=c_{1} \lambda_{1}(j \omega)^{i-2} \underline{v}_{1}(j \omega)+c_{2} \lambda_{2}(j \omega)^{i-2} \underline{v}_{2}(j \omega)$,

where $\underline{v}_{1}(j \omega), \underline{v}_{2}(j \omega)$ are the respective eigenvectors and $c_{1}, c_{2} \in$ $\mathbb{C}$ are constants such that in (24) we can write

$c_{1} \underline{v}_{1}(j \omega)+c_{2} \underline{v}_{2}(j \omega)=\begin{aligned} & 1 \\ & 1\end{aligned}$

at the particular value $\omega$. Furthermore, if we take the products

$$
\begin{aligned}
& \mathrm{M}(s) \begin{array}{l}
1 \\
1
\end{array}=\begin{array}{c}
\eta e^{\tau s} T+(1-\eta)\left(e^{\tau s}-1\right) T \\
\eta e^{\tau s} T+(1-\eta)\left(e^{\tau s}-1\right) T+1
\end{array} \\
& M(s) \begin{array}{c}
0 \\
1
\end{array}=\begin{array}{c}
(1-\eta)\left(e^{\tau s}-1\right) T \\
(1-\eta)\left(e^{\tau s}-1\right) T+1
\end{array},
\end{aligned}
$$

we see that $\left[\begin{array}{ll}1 & 1\end{array}\right]^{\top}$ cannot be an eigenvector for any $\omega$ and therefore the constants $c_{1}$ and $c_{2}$ in (55) belong to $\mathbb{C} \backslash\{0\}$. Similarly, the vector $\left[\begin{array}{ll}0 & 1\end{array}\right]^{\top}$ can only be an eigenvector if $e^{j \tau \omega}-1=0$ which shows that $v_{1,1}(j \omega)$ and $v_{2,1}(j \omega)$, the first components of the vectors $\underline{v}_{1}(j \omega)$ and $\underline{v}_{2}(j \omega)$ respectively, are not 0 at the frequencies considered. The result is that both eigenvalues in (54) will be contained in the expression for $F_{n, 1}$ whenever $e^{j \tau \omega}-1 \neq 0$. In other words, at frequencies such that $\rho(\mathrm{M}(j \omega))>1$

$\left|F_{n, 1}\right|=|S H|\left|c_{1} v_{1,1} \lambda_{1}^{n-2} v_{1}+c_{2} v_{2,1} \lambda_{2}^{n-2} v_{2}\right|$,

and recalling that at these frequencies $\left|\lambda_{1}\right|^{n-2} \rightarrow 0$ and $\left|\lambda_{2}\right|^{n-2} \rightarrow$ $\infty$ as $n \rightarrow \infty$ we can state that there is no $c \in \mathbb{R}$ such that $\left\|F_{n, 1}\right\|_{\infty} \leq c$ for all $n \in \mathbb{N}$.

The previous result does not require $\|T\|_{\infty}>1$ nor does it depend on the value $\eta$. We can see that this particular method for providing the followers with the leader state (alternative algorithm) leads to unavoidable string instability when there is time delay in the broadcast. 


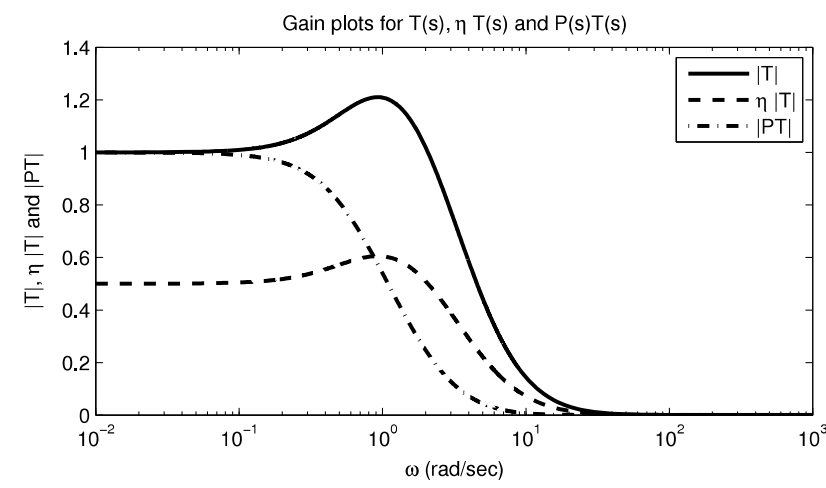

Fig. 2. Magnitude plots of $T, \eta T$ and $P T$ for the selected parameter values.
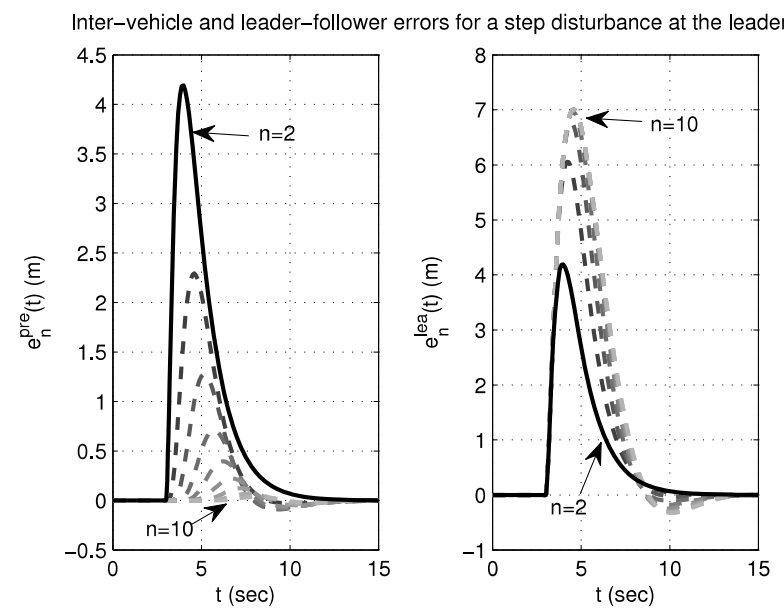

Fig. 3. Inter-vehicle and leader-follower errors for the leader-predecessor following scheme for a step disturbance of magnitude 10 at the leader. No time delay.

\section{Examples and simulations}

For all the examples we use

$$
\begin{aligned}
& H=\frac{1}{s(0.1 s+1)}, \quad K=\frac{2 s+1}{s(0.05 s+1)}, \quad K_{p}=\frac{1}{s(0.05 s+1)}, \\
& K_{v}=\frac{2}{s(0.05 s+1)}, \quad P=\frac{1}{2 s+1}, \quad \eta=0.5
\end{aligned}
$$

In Fig. 2 we can view the magnitude plots of $T, \eta T$ and $P T$ that yield from the selected parameters above. The reader can note that $K_{p}$ and $K_{v}$ are marginally stable and that $\|P T\|_{\infty} \leq 1$ with $P(0)=1$ (see Remark 4).

\subsection{Leader-predecessor following}

Simulations for the leader-predecessor following scheme illustrate the results stated in the previous sections. Under the absence of time delays in the communications it can be seen in Fig. 3 that the response of a step disturbance of magnitude 10 on the leader yields inter-vehicle errors that decrease along the string. Moreover, the steady state errors are 0 , which is consistent with our derivations. It is of no surprise to see that the leader-follower errors are also bounded with the string size increases.

Next, we consider multi-step string relay communications. For a time delay of $\tau=0.6$ (s) we see in Fig. 4 that the 0 DC gain of the inter-vehicle spacing error transfer functions is lost, as predicted
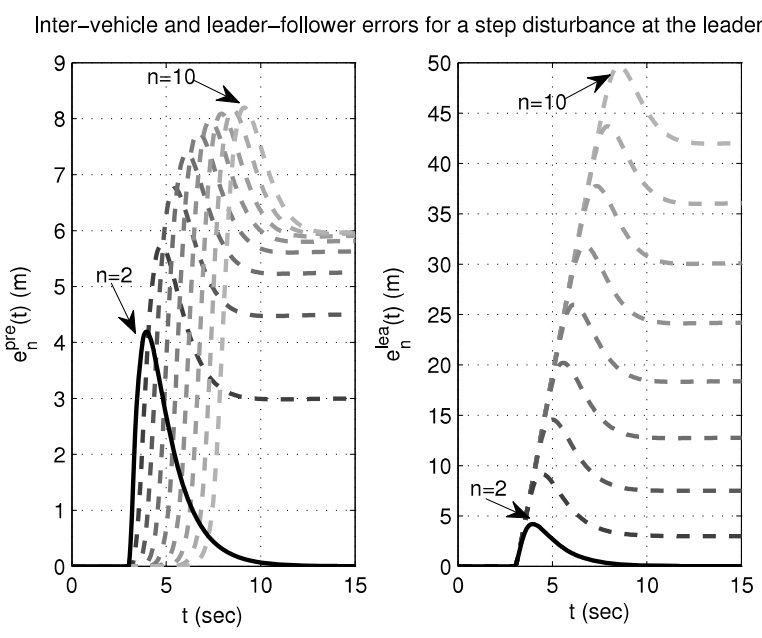

Fig. 4. Inter-vehicle and leader-follower errors for the leader-predecessor following scheme for a step disturbance of magnitude 10 at the leader. Multi-step string relay communications with $\tau=0.6$ (s).
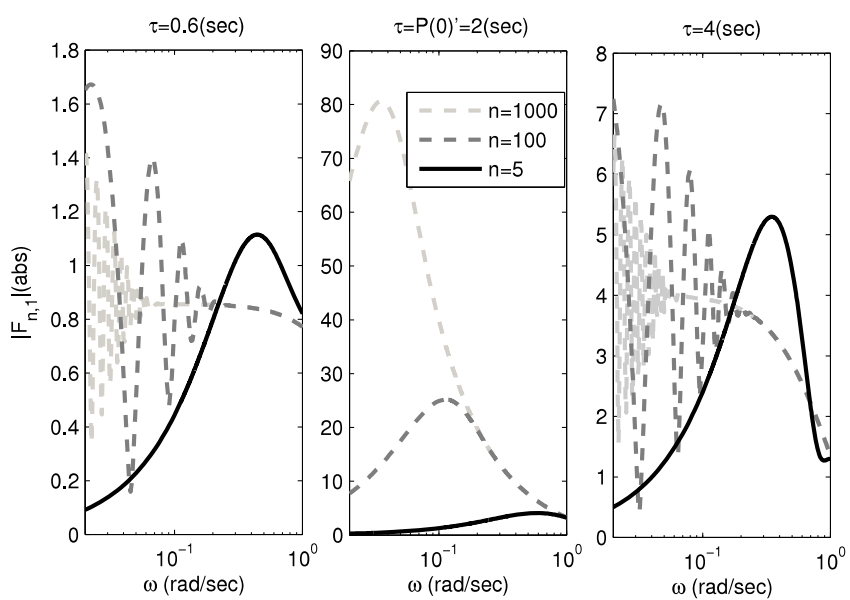

Fig. 5. Magnitude plots of $F_{n, 1}$ for $n=\{5,100,1000\}$ for the leader velocity tracking scheme. Multi-step string relay communications with $\tau=0.6$ (s) (string stable), $\tau=P^{\prime}(0)=2$ (s) (string unstable) and $\tau=4$ (s) (string stable).

by Theorem thm:distlead1relay, which in this case yields

$F_{n, 1}(0)=\tau \tilde{H}(0)\left(1-\eta^{n-2}\right)=0.6\left(1-0.5^{n-2}\right)$,

which tends to 0.6 as $n \rightarrow \infty$. Additionally we see the leaderfollower errors growing along the string, as predicted by Theorem 4.

\subsection{Leader velocity tracking}

For the velocity tracking scheme we compute some magnitude plots of $F_{n, 1}$ in the multi-step string relay communications. The first and third plots of Fig. 5 show that values of time delay different from the critical value (which in this case is $\tau=P^{\prime}(0)=2(\mathrm{~s})$ ), yield string stability for the predecessor error transfer functions. This is suggested by the fact $\left\|F_{1000,1}\right\|_{\infty} \leq\left\|F_{100,1}\right\|_{\infty}$ in both plots. On the contrary, for the critical time delay, the second plot of Fig. 5 shows a clear increase of $\left\|F_{n, 1}\right\|_{\infty}$ as $n$ grows. Finally, it can be seen that the bound on the magnitude peak increases with the value of $\tau$.

\subsection{Alternative algorithm: indirect leader state broadcast}

Finally, we consider the alternative algorithm for leader state broadcast. In Fig. 6 the step response of the predecessor errors is 


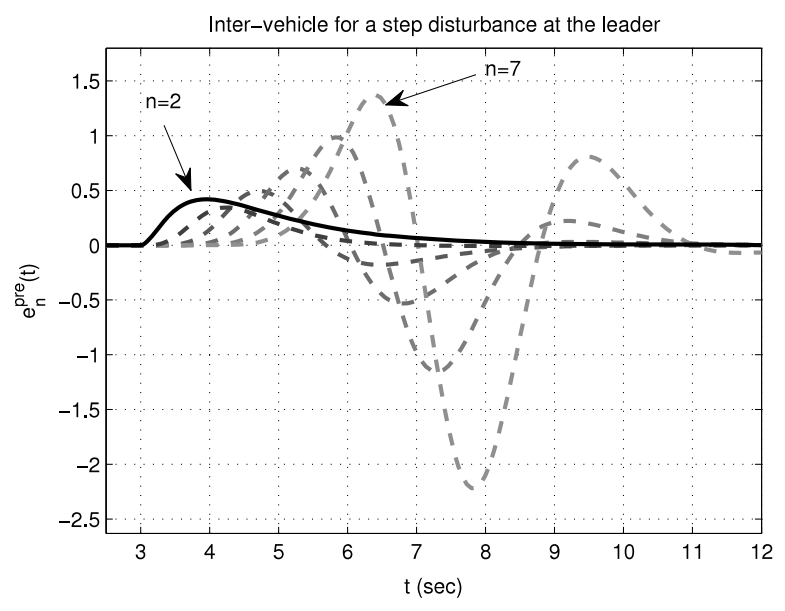

Fig. 6. Inter-vehicle errors of the alternative leader-predecessor algorithm for a step disturbance of magnitude 10 at the leader. Communications with $\tau=0.6$ (s).
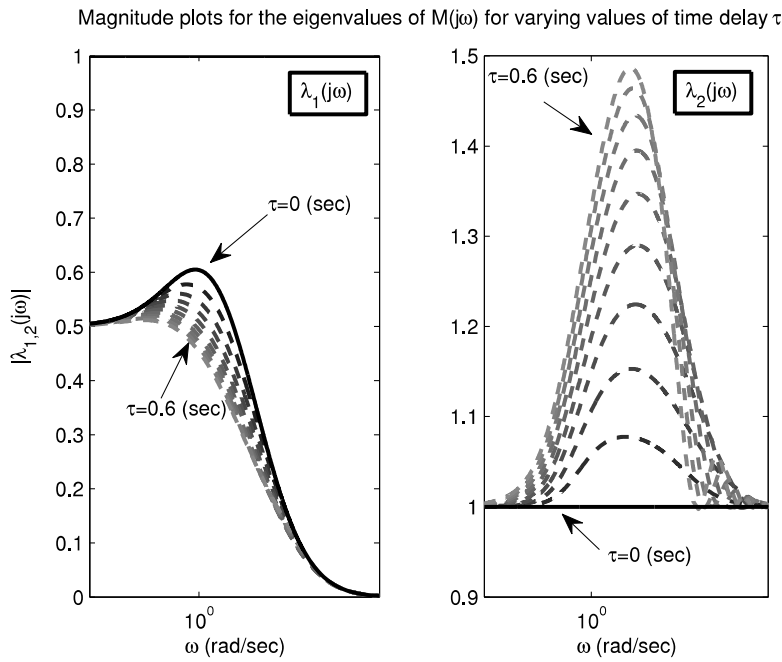

Fig. 7. Magnitude plots of the eigenvalues $\lambda_{1}(j \omega), \lambda_{2}(j \omega)$ of the matrix $M(j \omega)$ from the alternative algorithm for different amounts of time delay.

plotted for a time delay of $\tau=0.6(\mathrm{~s})$. It provides $0 \mathrm{DC}$ gain for the predecessor errors; however, string stability is lost under the presence of time delay. This is reflected by the increase in the peak response of the predecessor errors as the string length grows.

The magnitudes of the eigenvalues of the matrix $\mathrm{M}(j \omega)$ are plotted in Fig. 7 with the time delay as a varying parameter. It is clear that $\left|\lambda_{2}(j \omega)\right|$ takes values greater than 1 for $\tau>0$ as predicted by Lemma 2 .

\section{Conclusion}

In this paper, we studied three leader tracking schemes for formation control of vehicle strings. In particular we provided the inter-vehicle and leader-follower spacings dynamics resulting from the use of the three schemes, including possible time delays. We have shown that these architectures provide tight formations and string stability if the leader state is received instantaneously by the followers. However, if time delays occur in the leader state transmission only one of the schemes, namely leader velocity tracking, is able to maintain a tight formation while achieving string stability in the predecessor errors. An immediate extension to the present work is to obtain conditions for the existence and a methodology for the design of the controllers $K_{p}$ and $K_{v}$ in (6) from the velocity tracking scheme.
As a final remark we note that the presence of time delays in the broadcast of the leader state has different effects for each architecture, despite the fact that the three share some similarities. In particular, the alternative algorithm for indirect leader state broadcast becomes string unstable for any amount of time delay, independent of the vehicle dynamics or the design of the local controllers. This fact suggests that the way in which the leader state is sent to the followers and/or used by them plays a major role in the capability of a leader tracking architecture for formation control to achieve string stability.

\section{Acknowledgements}

The authors would like to thank the Editor and anonymous reviewers for their helpful suggestions, which helped to improve the quality of the manuscript. They also gratefully acknowledge the helpful advice of Professor Fabian Wirth in relation to the proof of Lemma 2.

\section{Appendix A. Proof of Theorem 3}

(1) The proof is similar to part (1) of Theorems 1 and 2.

(2) For dynamic $P$, such that $P(0)=1$ we can rewrite (38) as

$$
\begin{aligned}
F_{n, 1}= & H(1-P)(P T)^{n-n_{r}-1} T+(P T)^{n-2} H S \\
& -e^{-\tau S} H(1-P)(P T)^{n-n_{r}-1} T .
\end{aligned}
$$

We have that $S H$ and $H(1-P)$ are bounded at $s=0$ since the integrator of $H$ is cancelled by the zero at $s=0$ of $S$ and $(1-P)$ respectively. Now, Proposition 1 can be applied to every term of (A.1) and $\left\{F_{n, 1}\right\}$ is string stable.

For $P=\eta \in(0,1)$, we can once again rewrite $F_{n, 1}$

$F_{n, 1}=(\eta T)^{n-2} H S+\left(1-e^{-\tau s}\right) H \frac{(1-\eta)}{\eta}(\eta T)^{n-n_{r}}$

The limit

$\lim _{s \rightarrow 0}\left(1-e^{-\tau s}\right) H=\lim _{s \rightarrow 0} \frac{\left(1-e^{-\tau s}\right)}{s} \tilde{H}=\tilde{H}(0) \tau$,

shows that $\left\|\left(1-e^{-\tau s}\right) H\right\|_{\infty}$ is well defined and depends on the value of $\tau$. Proposition 1 can now be applied since $\|\eta T\|_{\infty} \leq 1$ and $\|S H\|_{\infty}$ and $\left\|\left(1-e^{-\tau s}\right) H(1-\eta) / \eta\right\|_{\infty}$ are well defined. Thus the sequence $\left\{F_{n, 1}\right\}$ is string stable when $P=\eta \in(0,1)$.

(3) For the leader error transfer function $F_{n, 1}$ we focus our attention on the second term of (40), noting that the first is exactly $\mathrm{F}_{n, 1}$ of the perfect communication case in (35). Since we assume that $\|P T\|_{\infty} \leq 1$, and $P T \neq 1$ for $s=j \omega, \omega>0$, the norm $\|(1-$ $P) /(1-P T) \|_{\infty}$ is well defined. Recalling that $\left\|\left(1-e^{-\tau s}\right) H\right\|_{\infty}<$ $\infty$, we have that $\left\|\left(1-e^{-\tau s}\right) H(1-P) /(1-P T)\right\|_{\infty}<\infty$ and Proposition 1 shows that the sequence $\left\{F_{n, 1}\right\}$ is string stable.

\section{Appendix B. Proof of Theorem 4}

(1) First, we will show that the term $(1-P T)(P T)^{n-3}-(1-$ $P) e^{-\tau(n-2) s}$ inside of the brackets in (42) has two zeros at $s=0$ when $P$ is dynamic. Differentiating we obtain

$$
\begin{aligned}
& (1-P T)(P T)^{n-3}-(1-P) e^{-\tau(n-2) s}{ }^{\prime} \\
& =(n-3)(1-P T)(P T)^{n-4}(P T)^{\prime}-(P T)^{\prime}(P T)^{n-3} \\
& \quad+P^{\prime} e^{-\tau(n-2) s}+\tau(n-2)(1-P) e^{-\tau(n-2) s} .
\end{aligned}
$$

Since $S$ has two zeros at the origin we have that $T^{\prime}(0)=$ $-S^{\prime}(0)=0$ which in turn implies $(P T)^{\prime}(0)=P^{\prime}(0)$. Recalling that 
$P(0)=1$ we have that

$$
\begin{aligned}
& \frac{d}{d s}(1-P T)(P T)^{n-3}-(1-P) e^{-\tau(n-2) s} \quad s=0 \\
& \quad=-P^{\prime}(0)+P^{\prime}(0)=0,
\end{aligned}
$$

and, due to the fact that the third term inside the brackets of (42) has two zeros at $s=0$, we have that $F_{n, 1}(0)=0$ and consequently $\mathrm{F}_{n, 1}(0)$.

The DC gain when $P=\eta \in(0,1)$ can be obtained in a similar fashion.

(2) The first two terms inside the brackets of (42) satisfy the hypothesis of Proposition 1 for any $P$. The third term requires further study. For dynamic $P$, such that $P^{\prime}(0) \neq-\tau$, we have the limit

$\lim _{s \rightarrow 0} \frac{1-P T}{P T-e^{-\tau s}}=-\frac{P^{\prime}(0)}{P^{\prime}(0)+\tau}$.

Also, $P T-e^{-\tau s}$ has only one zero at $s=0$ and no other zeros for $s=j \omega, \omega>0$. Therefore $\left\|(1-P T)\left(P T-e^{-\tau j \omega}\right)\right\|_{\infty}<\infty$ and Proposition 1 implies string stability of $\left\{F_{n, 1}\right\}$.

Remark 6. If the conditions for part (2) of Theorem 4 are not met we have string instability. If $P T-e^{-\tau s}$ has a zero at $s=j \omega_{c}$, that is $P\left(j \omega_{c}\right) T\left(j \omega_{c}\right)=e^{j \tau \omega_{c}}$, string stability is lost since we can only claim

$\lim _{s \rightarrow j \omega_{c}} \frac{(P T)^{n-3}-e^{-(n-3) \tau s}}{P T-e^{-\tau s}}=n-3$.

If $\tau=-P^{\prime}(0)$, that is, the critical amount of time delay, string instability arises once again. The limit

$\lim _{s \rightarrow 0} \frac{(P T)^{n-3}-e^{-(n-3) \tau s}}{P T-e^{-\tau s}}=n-3$,

yields a magnitude peak of the last term inside the brackets of (42) that grows with $n$.

(3) If $P$ is dynamic, we note that for $\tau>0,1-e^{-(n-1) \tau s}$ has zeros at arbitrarily low values of $\omega$ for increasing $n$ and $e^{-\tau s}-P T$ will not cancel them in (43). Now, it remains to compute the limit for the first term of (43)

$$
\lim _{s \rightarrow 0} H \frac{\left(1-(P T)^{n-1}\right)\left(e^{-\tau s}-T\right)}{e^{-\tau s}-P T}=\frac{(1-n) \tau \tilde{H}(0) P^{\prime}(0)}{\tau+P^{\prime}(0)} .
$$

This limit shows that for small frequencies $\left|F_{n, 1}(j \omega)\right|$ will grow unbounded with $n$, regardless of the selection of the dynamic $P$ and the value of $\tau>0$. Moreover, we will have an even worse response if $\tau$ gets closer to $-P^{\prime}(0)$, which is expected from the analysis carried out for $F_{n, 1}$.

For $P=\eta \in(0,1)$, the $\mathrm{DC}$ gain of $\mathrm{F}_{n, 1}$ is

$$
\begin{aligned}
\mathrm{F}_{n, 1}(0) & ={ }_{i=2}^{n} \tau \tilde{H}(0)\left(1-\eta^{i-2}\right) \\
& =\tau \tilde{H}(0) \quad n-1-\frac{1-\eta^{n-1}}{1-\eta} .
\end{aligned}
$$

This value grows with the platoon size $n$ and implies the string instability of $\left\{F_{n, 1}\right\}$.

\section{References}

Bamieh, B., Jovanovic, M. R., Mitra, P., \& Patterson, S. (2012). Coherence in large-scale networks: dimension-dependent limitations of local feedback. IEEE Transactions on Automatic Control, 57(9), 2235-2249.

Barooah, P., \& Hespanha, J. P. (2005). Error amplification and disturbance propagation in vehicle strings with decentralized linear control. In 44th IEEE conference on decision and control, 2005 and 2005 European control conference, CDC-ECC'05 (pp. 4964-4969). IEEE.
Barooah, P., Mehta, P. G., \& Hespanha, J. P. (2009). Mistuning-based control design to improve closed-loop stability margin of vehicular platoons. IEEE Transactions on Automatic Control, 54(9), 2100-2113.

Chien, C.C., \& Ioannou, P. (1992). Automatic vehicle-following. In American control Conference (pp. 1748-1752). June 1992.

Chu, K. C. (1974). Decentralized control of high-speed vehicular strings. Transportation Science, 8(4), 361-384.

Hao, H., Yin, H., \& Kan, Z. (2012). On the robustness of large 1-d network of double integrator agents. In American control conference ACC (pp. 6059-6064).

Hedrick, J. K., Tomizuka, M., \& Varaiya, P. (1994). Control issues in automated highway systems. IEEE Control Systems, 14(6), 21-32.

Horn, R., \& Johnson, C. (1999). Matrix analysis. Cambridge University Press.

Jovanovic, M. R., \& Bamieh, B. (2005). On the ill-posedness of certain vehicular platoon control problems. IEEE Transactions on Automatic Control, 50(9), 1307-1321.

Khatir, M., \& Davidson, E. (2004). Bounded stability and eventual string stability of a large platoon of vehicles using non-identical controllers. In 43rd IEEE conference on decision and control, CDC. Vol. 1 (pp. 1111-1116).

Klinge, S., \& Middleton, R. (2009). Time headway requirements for string stability of homogeneous linear unidirectionally connected systems. In Decision and control, 2009 held jointly with the 2009 28th Chinese control conference. CDC/CCC 2009. Proceedings of the 48th IEEE conference on. (pp. 1992-1997)

Lestas, I., \& Vinnicombe, G. (2007). Scalability in heterogeneous vehicle platoons. In American control conference, ACC'07 (pp. 4678-4683).

Levine, W., \& Athans, M. (1966). On the optimal error regulation of a string of moving vehicles. IEEE Transactions on Automatic Control, 11(3), 355-361.

Lin, F., Fardad, M., \& Jovanovic, M. R. (2012). Optimal control of vehicular formations with nearest neighbor interactions. IEEE Transactions on Automatic Control, 57(9), 2203-2218.

Liu, X., Goldsmith, A., Mahal, S., \& Hedrick, J. (2001). Effects of communication delay on string stability in vehicle platoons. In Proceedings on intelligent transportation systems, 2001 (pp. 625-630). IEEE.

Middleton, R. (1991). Trade-offs in linear control system design. Automatica, 27(2), 281-292.

Middleton, R., \& Braslavsky, J. (2010). String instability in classes of linear time invariant formation control with limited communication range. IEEE Transactions on Automatic Control, 55(7), 1519-1530.

Seiler, P., Pant, A., \& Hedrick, K. (2004). Disturbance propagaton in vehicle strings. IEEE Transactions on Automatic Control, 49(10), 1835-1841.

Shaw, E., \& Hedrick, J. (2007). String stability analysis for heterogeneous vehicle strings. In American control conference, ACC'07 (pp. 3118-3125).

Swaroop, D., \& Hedrick, J. (1996). String stability of interconnected systems. IEEE Transactions on Automatic Control, 41(3), 349-357.

Swaroop, D., Hedrick, J., Chien, C. C., \& Ioannou, P. (1994). A comparision of spacing and headway control laws for automatically controlled vehicles 1. Vehicle System Dynamics, 23(1), 597-625.

Xiao, L., Gao, F., \& Wang, J. (2009). On scalability of platoon of automated vehicles for leader-predecessor information framework. In Intelligent vehicles symposium, 2009 (pp. 1103-1108). IEEE.

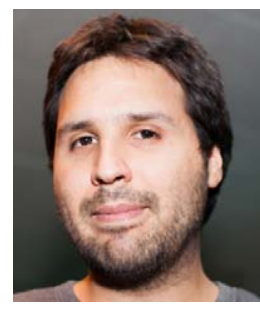

Andrés A. Peters was born in Coyhaique, Chile, in 1983. He obtained his Ingeniero Cicil Electrónico and Magíster en Igeniería Electrónica degrees from the Universidad Técnica Federico Santa María of Valparaíso, Chile in 2007. He is currently pursuing a Ph.D. degree at the Hamilton Institute from the National University of Ireland Maynooth. His research interests include performance bounds for optimal control of linear systems and string stability in formation control of vehicle platoons.

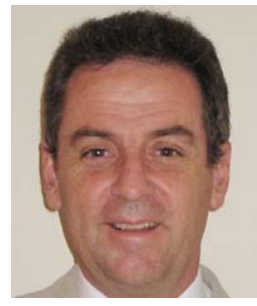

Richard H. Middleton was born on 10th December 1961 in Newcastle Australia. He received his B.Sc. (1983), B.Eng. (Hons-I) (1984) and Ph.D. (1987) from the University of Newcastle, Australia. He has had visiting appointments at the University of Illinois at Urbana-Champaign, the University of Michigan and the Hamilton Institute (National University of Ireland Maynooth). In 1991 he was awarded the Australian Telecommunications and Electronics Research Board Outstanding Young Investigator award. In 1994, he was awarded the Royal Society of New South Wales Edgeworth-David Medal; he was elected to the grade of Fellow of the IEEE starting 1999, and received the M.A. Sargent Award from 
the Electrical College of Engineers Australia in 2004. He has served as an associate editor of the IEEE Transactions on Automatic Control, the IEEE Transactions on Control System Technology, and Automatica, as the Head of the Department of Electrica and Computer Engineering at the University of Newcastle, as a panel member and sub panel chair for the Australian Research Council, as the Vice President - Member Activities and also as the Vice President - Conference Activities of the IEEE Control Systems Society, as the Director of the ARC Centre for Complex Dynamic Systems and Control, and, as an Associate Editor at Large and Senior Editor for the IEEE Transactions on Automatic Control. He is currently the director of the University of Newcastle Priority Research Centre for Complex Dynamic Systems and Control. His research interests include a broad range of Control Systems Theory and Applications, including Systems Biology with applications to Parkinson's Disease and HIV Dynamics.

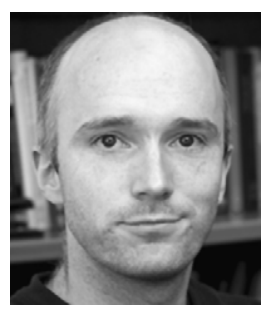

Oliver Mason studied mathematics at Trinity College Dublin (TCD), and was awarded a first class honours degree and a gold medal in his final examinations in 1995. He won several prizes as an undergraduate, including the Townsend Exhibition, Rowe Prize, Minchin Prize, The Lloyd Exhibition and was elected a foundation scholar of the college in 1993. He obtained an M.Sc. degree in mathematics by research from TCD in 1998 and in 2004 a Ph.D. on the stability of switched linear systems from the National University of Ireland Maynooth (NUIM). Currently, his major research interests include stability theory, applications of the max algebra to decision theory and privacy for online social networks and collaborative filtering systems. 